\title{
Recuperação avançada de petróleo associada à captura e armazenamento de carbono
}

\author{
Advanced oil recovery associated with carbon capture and storage \\ Recuperación mejorada de petróleo asociada con captura y almacenamiento de carbono
}

Recebido: 10/12/2021 | Revisado: 15/12/2021 | Aceito: 22/12/2021 | Publicado: 04/01/2022

\author{
Douglas Peterson Munis da Silva \\ ORCID: https://orcid.org/0000-0002-9505-505X \\ Universidade Federal de Itajubá, Brasil \\ E-mail: douglasilva@unifei.edu.br \\ Caio Vinicius Ventura Catarina \\ ORCID: https://orcid.org/0000-0002-0789-1542 \\ Universidade Federal de Itajubá, Brasil \\ E-mail: d2017004143@unifei.edu.br \\ Eric Alberto Ocampo Batlle \\ ORCID: https://orcid.org/0000-0001-9590-3111 \\ Universidade Federal de Itajubá, Brasil \\ E-mail: ericocampo@unifei.edu.br
}

\begin{abstract}
Resumo
As técnicas de Recuperação Avançada de Óleo (EOR) têm um grande potencial na indústria do petróleo e gás. Entre as possibilidades para aumentar a vida útil dos reservatórios está a EOR atuando em conjunto com o uso do dióxido de carbono $\left(\mathrm{CO}_{2}\right)$ capturado de fontes antropogênicas e naturais. Diante dos elevados custos associados às novas explorações de óleo há um interesse crescente na busca pela recuperação do óleo remanescente, via técnicas avançadas ou terciárias de recuperação, como a EOR, explorando a disponibilidade de óleo contido nos reservatórios. A crescente preocupação com o uso de recursos não renováveis e promissores de gases de efeito estufa (GEE), como o $\mathrm{CO}_{2}$, sobretudo para geração de energia, desencadeou a busca por tecnologias para mitigar as emissões de GEE. Entre essas tecnologias destaca-se a Captura e Armazenamento de Carbono (CCS) que captura o $\mathrm{CO}_{2}$ de fontes antrópicas que é posteriormente utilizado como insumo na indústria ou armazenado em formações geológicas. A CSS, em conjunto com a EOR, direciona o uso do $\mathrm{CO}_{2}$ para aumentar a produção de petróleo concomitante ao seu armazenamento. Este trabalho, por meio de uma revisão da literatura, trouxe dados que demonstram como o uso do $\mathrm{CO}_{2}$ vem sendo utilizado amplamente para a EOR em explorações onshore de óleo, e enfrentando desafios para expansão de seu uso em campos offshore. Foi identificado que fontes naturais de $\mathrm{CO}_{2}$ são predominantes, ou seja, não há uma contribuição significativa para redução das emissões de GEE.

Palavras-chave: Dióxido de carbono; Recuperação avançada de petróleo; Captura e armazenamento de carbono; Reservatório; Injeção de gás; Gases de efeito estufa.
\end{abstract}

\begin{abstract}
Advanced Oil Recovery (EOR) techniques have enormous potential in the oil and gas industry. Among the possibilities to increase the useful life of the reservoirs is EOR acting in conjunction with the use of carbon dioxide $\left(\mathrm{CO}_{2}\right)$ captured from anthropogenic and natural sources. Given the high costs associated with new oil explorations, there is a growing interest in the search for the recovery of the remaining oil, via advanced or tertiary recovery techniques, such as EOR, exploring the availability of oil contained in the reservoirs. The growing concern with the use of non-renewable and promising resources of greenhouse gases (GHG), such as $\mathrm{CO}_{2}$, especially for energy generation, has triggered the search for technologies to mitigate GHG emissions. Among these technologies, the Carbon Capture and Storage (CCS) stands out, which captures $\mathrm{CO}_{2}$ from anthropogenic sources, and it is later used as an input in industry or stored in geological formations. CSS, in conjunction with EOR, directs the use of $\mathrm{CO}_{2}$ to increase oil production concurrently with its storage. This work, through a literature review, brought data that demonstrate how the use of $\mathrm{CO}_{2}$ has been widely used for EOR in onshore oil exploration, and facing challenges for the expansion of its use in offshore fields. It was identified that natural sources of $\mathrm{CO}_{2}$ are predominant, that is, there is no significant contribution to reducing GHG emissions.
\end{abstract}

Keywords: Carbon dioxide; Advanced oil recovery; Carbon capture and storage; Reservoir; Gas injection; Greenhouse gases.

\section{Resumen}

Las técnicas de recuperación mejorada de petróleo (EOR) tienen un gran potencial en la industria del petróleo y el gas. Entre las posibilidades de incrementar la vida útil de los embalses está el EOR actuando en conjunto con el uso de dióxido de carbono $\left(\mathrm{CO}_{2}\right)$ capturado de fuentes antropogénicas y naturales. Dados los altos costos asociados a las 
nuevas exploraciones petroleras, existe un interés creciente en la búsqueda de la recuperación del petróleo remanente, a través de técnicas de recuperación mejorada o terciarias, como EOR, explorando la disponibilidad de petróleo contenido en los reservorios. La creciente preocupación por el uso de recursos no renovables y prometedores de gases de efecto invernadero (GEI), como el $\mathrm{CO}_{2}$, especialmente para la generación de energía, ha desencadenado la búsqueda de tecnologías para mitigar las emisiones de GEI. Entre estas tecnologías, se destaca la Captura y Almacenamiento de Carbono (CCS), que captura $\mathrm{CO}_{2}$ de fuentes antropogénicas y luego es utilizado como insumo en la industria o almacenado en formaciones geológicas. CSS, junto con EOR, dirige el uso de $\mathrm{CO}_{2}$ para aumentar la producción de petróleo al mismo tiempo que su almacenamiento. Este trabajo, a través de una revisión de la literatura, arrojó datos que demuestran cómo el uso de $\mathrm{CO}_{2}$ ha sido ampliamente utilizado para EOR en la exploración de petróleo en tierra y enfrentando desafíos para la expansión de su uso en campos costa afuera. Se identificó que las fuentes naturales de $\mathrm{CO}_{2}$ son predominantes, es decir, no hay un aporte significativo a la reducción de emisiones de GEI.

Palabras clave: Dióxido de carbono; Recuperación mejorada de petróleo; Captura y almacenamiento de carbono; Depósito; Inyección de gas; Gases de efecto invernadero.

\section{Introdução}

O petróleo e seus derivados permanecem sendo um recurso essencial para as atividades humanas. Esse recurso possibilitou o desenvolvimento da sociedade moderna. Sua produção e uso continuam em crescimento (Ritchie \& Roser, 2020), apesar de recomendações para um uso mais sustentável em face às mudanças climáticas.

Preocupações sobre as emissões de gases de efeito estufa (GEE) oriundos, substancialmente, do uso de fontes fósseis, como petróleo, têm suscitado o interesse em tecnologias que visam mitigar as emissões de GEE, principalmente no setor de energia. No rol dessas tecnologias, o interesse pela técnica de Recuperação Avançada de Petróleo, fazendo uso da injeção de $\mathrm{CO}_{2}$ (Enhanced Oil Recovery with $\mathrm{CO}_{2}-\mathrm{CO}_{2}$-EOR), tem aumentado e vai além das questões climáticas, uma vez que esse método permite prolongar a vida útil de um poço, aumentando a eficiência de produção do petróleo em muitos campos, sobretudo os maduros ou aqueles cuja produção já declinou consideravelmente.

Paralelo à técnica $\mathrm{CO}_{2}$-EOR está o processo de captura, uso ou armazenamento do carbono (Carbon Capture and Storage - CCS), que consiste na captura e purificação do $\mathrm{CO}_{2}$ oriundo de processos industriais, como da geração de energia, da indústria química, cimenteira e refinarias, ou seja, de setores de economia que encaram uma dificuldade na transição para uso de fontes renováveis ou de baixo teor de carbono.

No processo de $\mathrm{CCS}$, o $\mathrm{CO}_{2}$ é capturado, comprimido e transportado e pode ser utilizado como insumo na indústria ou armazenado em formações rochosas. Umas das alternativas para o uso do $\mathrm{CO}_{2}$ é o aumento da eficiência de extração de petróleo, que, além de contribuir para extração do petróleo remanescente, pode, em certas condições, permanecer aprisionado no poço.

Essas duas técnicas, CCS e EOR- $\mathrm{CO}_{2}$, atuando em conjunto, podem promover a redução das emissões de GEE e prolongar a vida útil dos reservatórios já maduros, aumentando a eficiência de recuperação do petróleo.

Assim, esse trabalho se propôs a apresentar um panorama geral dessas técnicas apresentando dois casos brasileiros de sucesso. O artigo se inicia descrevendo sobre o CCS; seguindo de uma descrição da técnica de EOR e, por fim, apresenta casos no Brasil.

\section{Metodologia}

O artigo que se segue está assentado em uma revisão da literatura. As referências aqui citadas foram obtidas a partir de plataformas de pesquisas, como o "Google Acadêmico" e o "Scopus", buscando por trabalhos que associam a técnica avançada de recuperação de petróleo à tecnologia de captura e armazenamento de carbono. A pesquisa foi delimitada pelas palavras chaves, redigidas na língua inglesa e português "EOR", "Enhanced oil recovery ou Recuperação Avançada de Petróleo", "CCS”, “Carbon Capture and Storage ou Captura, Armazenamento de Carbono". 
A metodologia, conhecida como "snowballing", que consiste na pesquisa das referências citadas pelos trabalhos encontrados, também foi utilizada.

\section{Captura, Uso e Armazenamento de Carbono}

Diante do crescimento global da produção e consumo de combustíveis fósseis, sobretudo no setor de energia, o aumento da concentração de $\mathrm{CO}_{2}$ e de outros GEE na atmosfera tem refletido em alterações climáticas que impactam negativamente a economia e o bem-estar humano, despertando o interesse em ações e planos cujo objetivo é mitigar ou reduzir as emissões de GEE.

Entre as técnicas mais promissoras para mitigar as emissões de $\mathrm{CO}_{2}$, sobretudo em setores da economia que enfrentam dificuldade para reduzir suas emissões, é o CCS, constituindo uma oportunidade para desacelerar os efeitos das mudanças climáticas (Perera et al., 2016).

A tecnologia de CCS tem se configurado, recentemente, econômica e ambientalmente viável. A CCS permite a captura de $\mathrm{CO}_{2}$ de fontes industriais ou da queima de combustíveis que é, posteriormente transportado, via navios ou dutos (pipelines), e utilizado como insumo ou armazenado em formações geológicas (Adu et al., 2019). A CCS é classificada em captura direta, quando o $\mathrm{CO}_{2}$ é capturado diretamente na fonte antes de ser emitido para atmosfera e em captura indireta, se o $\mathrm{CO}_{2}$ é capturado da atmosfera (Ravagnani et al., 2009).

Uma das alternativas para uso do $\mathrm{CO}_{2}$ capturado é a injeção deste gás em reservatórios de óleo em uma técnica conhecida como EOR-CO $\mathrm{CO}_{2}$. Essa técnica configura-se segura e efetiva para reduzir as emissões de GEE (Sweatman et al., 2011) e para aplicação na indústria de óleo e gás, visto que pode estender a vida útil dos reservatórios (Adu et al., 2019).

Em 2015, o $\mathrm{CO}_{2}$ injetado para a EOR era o segundo insumo mais usado para aumentar a eficiência de produção do poço, ficando atrás do uso da água de acordo com (Baena-Moreno et al., 2019). Para o CCS ser bem-sucedido, Ravagnani (2007) observa que a técnica precisa ser rentável e competitiva, fornecer armazenamento estável e a longo prazo, os impactos ambientais devem ser de pequena magnitude, minimizados ou evitados e o risco associado ao CCS precisa ser mínimo.

\section{Recuperação de Petróleo}

Um potencial reservatório de petróleo, após ser descoberto, começa a ser explorado. O estágio inicial de produção do óleo é conhecido por recuperação primária, que faz uso da energia primária existente no reservatório (observada na expansão de gases dissolvidos, variação do volume da rocha, diferença de pressão entre o reservatório e a superfície, influxo do aquífero); essa energia é suficiente para que o óleo se desloque naturalmente para superfície, cuja faixa de recuperação é entre 5-15\% do petróleo presente inicialmente no poço (Original Oil in Place - OOIP) (Abramova et al., 2014).

Entretanto, essa energia sofre depleção com o tempo, refletindo no decréscimo de pressão e redução da produtividade. Diante desse fato, devido aos custos crescentes associados à exploração de novos campos de petróleo, é conveniente investir em técnicas de recuperação secundária e posteriormente terciária, ou seja, antes de investir em novos desenvolvimentos e explorações, busca-se, primeiramente, recuperar o restante de óleo remanescente.

Em um segundo momento, quando a recuperação primária já se demonstra inefetiva, a secundária é utilizada, fazendo uso principalmente da injeção de água no reservatório para aumentar a pressão e a recuperação do óleo, alcançado uma eficiência em cerca de 33\% do OOIP (Perera et al., 2016).

O terceiro estágio é caracterizado por uma gama de técnicas que buscam extrair o óleo remanescente do poço; são técnicas de recuperação avançada de petróleo e podem ocorrer por via química, térmica e uso de gás (Alvarado \& Manrique, 2010). 
Estas técnicas ou métodos avançados incluem IOR (Improved Oil Recovery) que engloba o EOR e as atividades de gerenciamento e são aplicados quando os processos primário e secundário de recuperação se tornam ineficientes (Diniz, 2015).

Para a recuperação do petróleo existem basicamente duas técnicas que são baseadas na miscibilidade do fluido, propriedade muito importante a ser considerada antes da escolha do método de injeção mais adequado. A miscibilidade consiste na capacidade de mistura entre os fluidos, ou seja, a capacidade de tornar uma mistura homogênea ou de fase única.

A miscibilidade vai atuar principalmente no deslocamento do óleo, que, a depender da técnica e do fluido aplicados poderá ocorrer um deslocamento do tipo miscível ou do tipo imiscível. A escolha do tipo de técnica de miscibilidade dependerá das características do gás injetado, do reservatório, do óleo e da pressão mínima de miscibilidade (Minimum Miscibility Pressure - MMP) que é a pressão mínima requerida para se atingir a miscibilidade entre o gás injetado e o petróleo (Gozalpour et al., 2005). Ademais, os processos miscíveis são mais indicados para óleos leves (Hashemi Fath \& Pouranfard, 2014).

\subsection{Recuperação Avançada de Petróleo}

A recuperação avançada de petróleo, em inglês Enhanced oil recovery (EOR) consiste numa técnica que busca aumentar a eficiência de produção óleo, prolongando a vida útil do reservatório utilizado diversas vias: química, térmica, microbiológica ou pela injeção de gás (Gomes, 2019; Xu et al., 2010). As técnicas de EOR mais comuns estão apresentadas na Figura 1:

Figura 1 - Estágios de produção do petróleo.

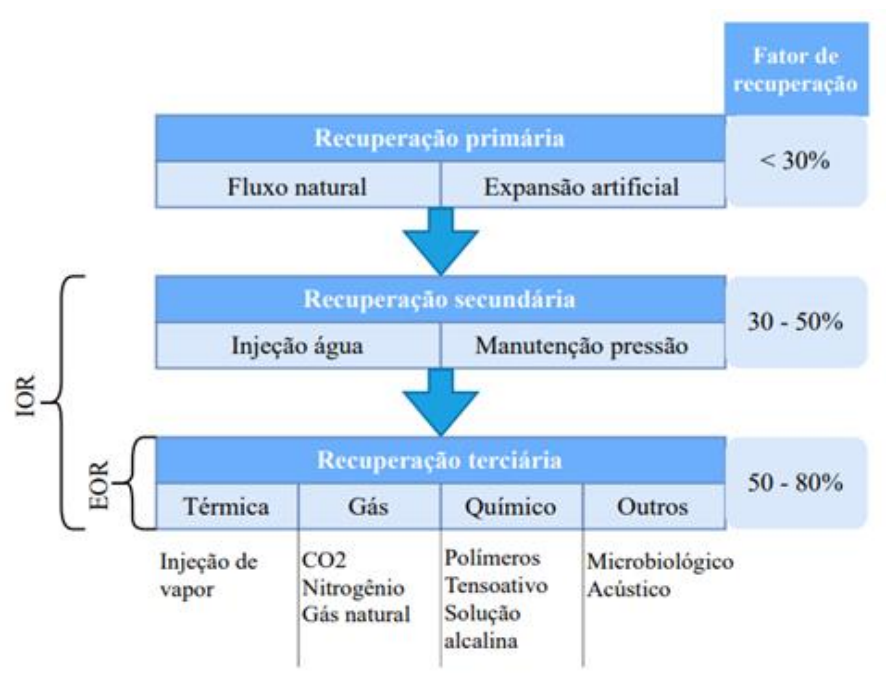

Fonte: Adaptado de Patel et al. (2018).

A escolha da via mais adequada dependerá das características do fluido, como viscosidade e densidade e das características do reservatório, como permeabilidade, porosidade, profundidade, pressão e temperatura (Khan \& Islam, 2007).

O método químico consiste na injeção direta de reagentes químicos ou misturados com água, com objetivo de limpar a zona de perfuração do poço para aumentar a eficácia de deslocamento dos fluidos ou ainda para reduzir a tensão superficial entre a superfície do reservatório e o óleo, reduzindo sua viscosidade e aumentando sua mobilidade. Esta técnica aumenta em até 35\% a recuperação do poço (Abramova et al., 2014; Perera et al., 2016). A recuperação química ainda é economicamente inviável em algumas aplicações, sobretudo devido ao elevado preço dos reagentes químicos. 
A recuperação térmica promove uma mudança nas características químicas e físicas do fluido, sendo indicada para os casos de maior dificuldade física e geológica de extração. Esta técnica permite a recuperação entre 30 e $58 \%$ do OOIP (Khan \& Islam, 2007).

A técnica mais comum de EOR utilizada atualmente é a técnica miscível ou injeção de gás que é introduzido no reservatório diminuindo a tensão superficial entre a água e o óleo, proporcionando um aumento entre 5 e $19 \%$ da recuperação comparado à recuperação secundária (Khan \& Islam, 2007).

\subsection{O uso do Dióxido de Carbono - $\mathrm{CO}_{2}$}

O uso do $\mathrm{CO}_{2}$-EOR já data de 1980 nos EUA, ou seja, não é uma tecnologia nova (Perera et al., 2016), entretanto a recente preocupação com os efeitos das mudanças climática, a transição para uma economia de baixo carbono e a possibilidade de aumentar a taxa de recuperação do petróleo (sobretudo em campos maduros) tem difundido a utilização do $\mathrm{CO}_{2}$-EOR (Adu et al., 2019).

Historicamente, o uso de fontes naturais de $\mathrm{CO}_{2}$ para a EOR era o mais comum, ou seja, não havia contribuição para reduzir as emissões antropogênicas de $\mathrm{CO}_{2}$ (Mattos, 2018). Todavia, esse cenário tem se alterado recentemente diante da existência de centenas de campo de petróleo aptos ao EOR e da disponibilidade, em quantidade e regularidade, de $\mathrm{CO}_{2}$ oriundo de diversas fontes antropogênicas, podendo resultar no armazenamento de $\mathrm{CO}_{2}$ que antes seria emitido para atmosfera (Pant et al., 2021) Pontua-se que a troca do $\mathrm{CO}_{2}$ natural pelo oriundo de fontes antropogênicas dependerá sobretudo dos custos de captura e dos incentivos políticos e fiscais (Cuéllar-Franca \& Azapagic, 2015).

$\mathrm{O} \mathrm{CO}_{2}$ introduzido no reservatório retorna, parcialmente, para a superfície em uma mistura de óleo, $\mathrm{CO}_{2} \mathrm{e}$ água, que é separada: o óleo é encaminhado para a refinaria e o $\mathrm{CO}_{2}$ separado, recomprimido e reinjetado no reservatório, ou seja, reciclado, evitando sua emissão para a atmosfera (Mattos, 2018). Contabiliza-se que cerca de $50 \%$ do $\mathrm{CO}_{2}$ injetado fica retido no reservatório, havendo, constantemente, a demanda por esse gás (Farajzadeh et al., 2020).

A opção do uso do $\mathrm{CO}_{2}$ para o EOR configura-se positiva em dois aspectos: a MMP geralmente é muito menor que a verificada com o uso de gás natural ou nitrogênio, em que o processo miscível atinge níveis de recuperação maiores que o imiscível (Verma, 2015). Um segundo aspecto é a flexibilidade do uso $\mathrm{CO}_{2}$ que pode ser utilizado em condições miscíveis e imiscíveis (Baena-Moreno et al., 2019).

Entretanto, somente em condições específicas o $\mathrm{CO}_{2}$ injetado pode permanecer armazenado no reservatório, atuando como uma forma geológica de armazenamento, proporcionando um meio de mitigar as emissões $\mathrm{CO}_{2}($ Cuéllar-Franca \& Azapagic, 2015).

Em 2014, 136 (cento de trinta seis) projetos de $\mathrm{CO}_{2}$-EOR estavam em operação nos EUA, injetando mais de três bilhões de pés cúbicos de $\mathrm{CO}_{2}\left(152 \mathrm{MtCO}_{2}\right)$ o que representa ainda uma fração muito pequena das emissões antropogênicas desse gás, visto que, grande parte do $\mathrm{CO}_{2}$ injetado é de origem natural, não contribuindo, portanto, para redução das emissões de GEE (Farajzadeh et al., 2020).

Salienta-se que aplicação dessas técnicas avançadas não necessariamente ocorrem apenas em poços maduros, podendo ser introduzidas em qualquer momento da vida produtiva do campo, entretanto são mais promissoras quando a razão entre a água e óleo produzido no campo se aproxima do limite econômico, havendo redução do lucro líquido (Diniz, 2015).

Assim, de acordo com Verma (2015), a EOR utilizando o $\mathrm{CO}_{2}$ pode, além de aumentar a recuperação do petróleo, atuar como uma técnica de armazenamento de $\mathrm{CO}_{2}$, refletindo na redução das emissões atmosféricas. 


\subsection{Desafios e potenciais da técnica $\mathrm{CO}_{2}-\mathrm{EOR}$}

Persistem desafios que dificultam a viabilidade e a expansão do $\mathrm{CO}_{2}$-EOR que captura $\mathrm{o} \mathrm{CO}_{2}$ de fontes antropogênicas, tanto para as explorações onshore como offshore. Esses desafios geram incertezas sobre a viabilidade e expansão da técnica $\mathrm{CO}_{2}$-EOR. Entre os desafios destacam-se os aspectos econômicos associados aos elevados custos de infraestrutura para captura e transporte do $\mathrm{CO}_{2}$, a ausência de um mercado regulado do $\mathrm{CO}_{2}$ e de políticas públicas de incentivo e fatores ambientais, como as condições geológicas e de segurança dos reservatórios (Sweatman et al., 2011).

As atividades de $\mathrm{CO}_{2}$-EOR onshore que capturam o carbono de fontes antropogênicas se beneficiam de fontes próximas de $\mathrm{CO}_{2}$, cenário diferentes das atividades offshore que enfrentam desafios logísticos, como indisponibilidade fontes regulares de $\mathrm{CO}_{2}$, de pipelines para o transporte do gás, possível ocorrência de problemas associados à corrosão e deposição e das dificuldades em monitorar o $\mathrm{CO}_{2}$ armazenado (Gozalpour et al., 2005). Em vias de contornar essas dificuldades, alguns projetos offshore utilizam do $\mathrm{CO}_{2}$ de reservas naturais de gás associado à exploração do óleo para as técnicas de EOR. Entretanto, é importante destacar que, nesses casos, devido à origem do $\mathrm{CO}_{2}$ não ser antropogênico, não se verifica uma redução das emissões de GEE.

$\mathrm{O}$ uso do método de $\mathrm{CO}_{2}-\mathrm{EOR}$, apesar de ter ganhado sustentação ao longo dos anos, ainda não é favorável economicamente, principalmente em condições offshore, visto que muitos operadores ainda não usam essa tecnologia em seus reservatórios. Relativo aos custos, Sweatman (2011) identificou que a parte mais custosa referia-se à captura e compressão do gás, seguida do armazenamento e por último o transporte.

A questão principal que induz a esse evento é o imaturo mercado de carbono que ainda carrega consigo inconsistências, falta de regularidade e de disponibilidade, sendo necessário que haja uma maturação dos sistemas de CCS, bem como uma imersão bem-sucedida no mercado, para que a demanda $\mathrm{CO}_{2}$ seja suprida (Sweatman et al., 2011).

\section{5. $\mathrm{CO}_{2}$ - EOR no Brasil}

No Brasil, o uso do $\mathrm{CO}_{2}$ para a EOR já vem sendo empregado há algum tempo na Região Nordeste, na Bacia do Recôncavo, em campos onshore, e mais recentemente, com a descoberta do pré-sal, no Campo de Lula.

\subsection{Campo de Lula}

O Campo de Lula, localizado na Região Sudeste, foi descoberto em 2006 na área conhecida como pré-Sal da Bacia de Santos. Os reservatórios de óleo estão localizados a uma profundidade de cerca de 2200 metros e a uma distância média de 230 quilômetros da costa. As reservas de óleo são estimadas entre 5 e 8 milhões de barris. Uma característica da exploração do óleo nesse campo é a parcela do $\mathrm{CO}_{2}$ associada à produção do óleo, que está entre 8 e 5\% (Eide et al., 2019).

Entre as dificuldades para exploração dessa área, a presença de um percentual considerável de $\mathrm{CO}_{2}$ foi substancial para que a aplicação técnica do $\mathrm{CO}_{2}$-EOR fosse considerada já no início da exploração.

Apesar dos desafios do $\mathrm{CO}_{2}$-EOR em explorações offshore, a presença de um percentual $\mathrm{CO}_{2}$ associado à produção do óleo demonstrou-se uma estratégica econômica e ambientalmente viável: o $\mathrm{CO}_{2}$ foi utilizado para aumentar a eficiência de recuperação do óleo paralelo à decisão de não emitir o $\mathrm{CO}_{2}$ para a atmosfera. As características do reservatório, como a temperatura entre 60 e $70^{\circ} \mathrm{C}$ e a alta pressão permitem a injeção miscível de $\mathrm{CO}_{2}$ (Eide et al., 2019; Kuuskraa \& Malone, 2016). 


\subsection{Bacia do Recôncavo}

A Bacia do Recôncavo está localizada em terra na Região Nordeste do Brasil, no Estado da Bahia, onde há mais de 80 campos de petróleo, sua exploração já acontece há mais de 70 anos, ou seja, os campos já são maduros e, por essa razão, a partir do início da década de 1990 começou a ser utilizado o $\mathrm{CO}_{2}$ para recuperação do óleo residual. A partir da década de 1960 a exploração do óleo já se demonstrava economicamente inviável (Dino \& Gallo, 2009).

$\mathrm{O} \mathrm{CO}_{2}$ era obtido de uma indústria de fertilizantes localizada a $75 \mathrm{~km}$ do campo de exploração, precisando ser comprimido e transportado por dutos. O gás era injetado alternativamente com água, e entre os anos de 1991 e 2005 , cerca de 600 mil toneladas de $\mathrm{CO}_{2}$ foram injetadas nesse campo possibilitando a produção de cerca de $1 \mathrm{~m}^{3}$ adicional de óleo a partir da injeção de 2,58 toneladas de $\mathrm{CO}_{2}$, sendo que $50 \%$ do $\mathrm{CO}_{2}$ injetado retornava misturado ao petróleo extraído (Ravagnani, 2007)

Assim, o processo de $\mathrm{CO}_{2}$-EOR na Bacia do Recôncavo utilizava de duas técnicas em conjunto: a CCS e a EOR, que, conforme ilustrado pela Figura 2, possibilitou o aumento da recuperação do óleo concomitante ao armazenamento do $\mathrm{CO}_{2}$.

Figura 2 - Recuperação do óleo na Bacia do Recôncavo.

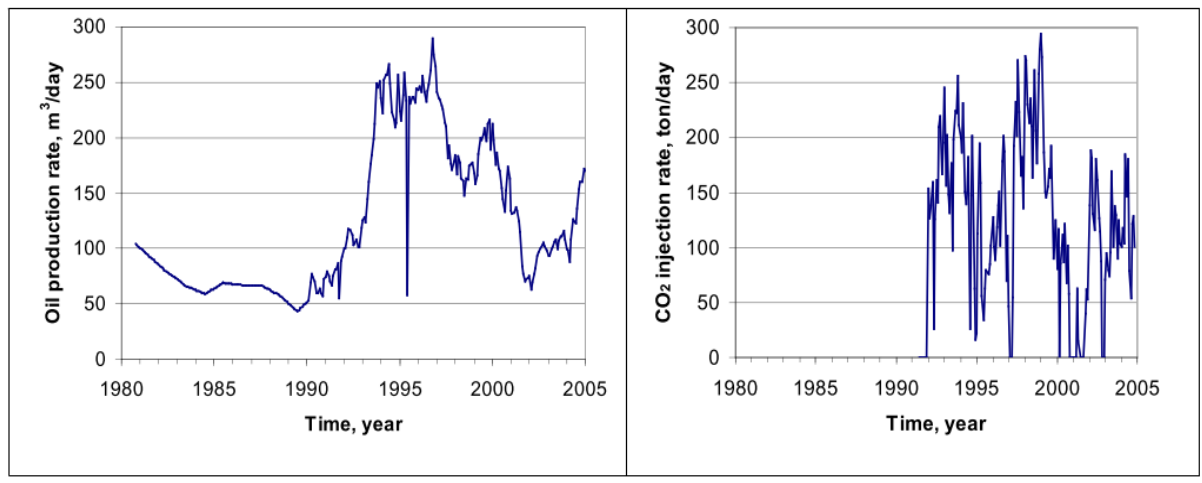

Fonte: Dino e Gallo (2009).

\section{Considerações Finais}

A técnica $\mathrm{CO}_{2}$-EOR associada a CCS oferece uma alternativa para aumentar a recuperação de óleo e armazenar o $\mathrm{CO}_{2}$ em formações geológicas. Entretanto, muitos projetos de EOR continuam utilizando o $\mathrm{CO}_{2}$ de origem natural, não contribuindo, portanto, para a redução das emissões de GEE.

A opção pelas fontes naturais de $\mathrm{CO}_{2}$ deu-se pela diante da carência de fontes antropogênicas que fornecessem $\mathrm{CO}_{2}$ em quantidade e regularidade necessários para a indústria de óleo e gás, por questões de inviabilidade econômica da captura direta de $\mathrm{CO}_{2}$, pelos custos associados à purificação do gás e pelo transporte e as atividades de armazenamento e monitoramento do $\mathrm{CO}_{2}$.

Todavia, incentivos e políticas públicas podem inverter esse cenário de desafios econômicos. O reconhecimento que a atuação conjunta desses métodos pode gerar efeitos que vão além da esfera econômica pode despertar o interesse por essas tecnologias em uma economia que, a passos lentos, está se descarbonizando. A opção pela injeção de $\mathrm{CO}_{2}$ demonstra-se mais eficaz para recuperação avançada de petróleo, devido a sua alta eficiência de deslocamento, e ao seu potencial para benefícios ambientais.

Através de um panorama otimista é muito provável que em curto prazo as tecnologias de captura de $\mathrm{CO}_{2}$ avancem e se solidifiquem no mercado, amenizando assim seus custos, bem como o aprimoramento das tecnologias de recuperação avançada. 
Os autores pontuam, por fim, que pesquisas futuras venham a se debruçar sobre novas oportunidades para captura de $\mathrm{CO}_{2}$ no Brasil, avaliando o potencial econômico, técnico e ambiental da $\mathrm{CO}_{2}$-EOR. Diante disso, faz-se necessário o aprofundamento no assunto por meio de uma análise substancial da evolução da tecnologia de CCS. A evolução da CCS em conjunto com a EOR pode promover a redução dos custos nos próximos anos, ampliando as possibilidades de aplicação dessas tecnologias.

\section{Agradecimentos}

Os autores agradecem à Agência Nacional do Petróleo pelo financiamento da pesquisa pelo PRH-ANP n46.1.

\section{Referências}

Abramova, A., Abramov, V., Kuleshov, S., \& Timashev, E. (2014). Analysis of the Modern Methods for Enhanced Oil Recovery. Energy, 3, 118-148. https://doi.org/10.13140/2.1.2709.4726

Adu, E., Zhang, Y., \& Liu, D. (2019). Current situation of carbon dioxide capture, storage, and enhanced oil recovery in the oil and gas industry. The Canadian Journal of Chemical Engineering, 97(5), 1048-1076. https://doi.org/10.1002/CJCE.23393

Alvarado, V., \& Manrique, E. (2010). Enhanced Oil Recovery: An Update Review. Energies, 3(9), 1529-1575. https://doi.org/10.3390/en3091529

Baena-Moreno, F. M., Rodríguez-Galán, M., Vega, F., Alonso-Fariñas, B., Vilches Arenas, L. F., \& Navarrete, B. (2019). Carbon capture and utilization technologies: a literature review and recent advances. Energy Sources, Part A: Recovery, Utilization and Environmental Effects, 41(12), 1403-1433. https://doi.org/10.1080/15567036.2018.1548518

Cuéllar-Franca, R. M., \& Azapagic, A. (2015). Carbon capture, storage and utilisation technologies: A critical analysis and comparison of their life cycle environmental impacts. Journal of CO2 Utilization, 9, 82-102. https://doi.org/10.1016/j.jcou.2014.12.00

Diniz, A. A. R. (2015). Estudo da injeção de água e $\mathrm{CO}_{2}$ em reservatório carbonático de óleo leve [Tese de Doutorado, Universidade Federal do Rio Grande do Norte].

Dino, R., \& Gallo, Y. Le. (2009). CCS project in Recôncavo Basin. Energy Procedia, 1(1), 2005-2011. https://doi.org/10.1016/j.egypro.2009.01.261

Eide, L. I., Batum, M., Dixon, T., Elamin, Z., Graue, A., Hagen, S., Hovorka, S., Nazarian, B., Nøkleby, P. H., Olsen, G. I., Ringrose, P., \& Vieira, R. A. M. (2019). Enabling Large-Scale Carbon Capture, Utilisation, and Storage (CCUS) Using Offshore Carbon Dioxide $\left(\mathrm{CO}_{2}\right)$ Infrastructure Developments-A Review. Energies, 12(10), 1945. https://doi.org/10.3390/EN12101945

Farajzadeh, R., Eftekhari, A. A., Dafnomilis, G., Lake, L. W., \& Bruining, J. (2020). On the sustainability of $\mathrm{CO}_{2}$ storage through $\mathrm{CO}_{2}-$ Enhanced oil recovery. Applied Energy, 261, 114467. https://doi.org/10.1016/j.apenergy.2019.114467

Gomes, D. M. (2019). Recuperação de reservatório por injeção de dióxido de carbono: análise de estudo de casos [Monografia de Graduação, Universidade Federal do Amazonas].

Gozalpour, F., Ren, S. R., \& Tohidi, B. (2005). $\mathrm{CO}_{2}$ EOR and Storage in Oil Reservoirs. Oil and Gas Science and Technology, 60(3), 537-546. https://doi.org/10.1007/s10750-018-3759-9

Hashemi Fath, A., \& Pouranfard, A. R. (2014). Evaluation of miscible and immiscible $\mathrm{CO}_{2}$ injection in one of the Iranian oil fields. Egyptian Journal of Petroleum, 23(3), 255-270. https://doi.org/10.1016/J.EJPE.2014.08.002

Khan, M. I., \& Islam, M. R. (2007). Enhanced Oil Recovery (EOR) Operations. In M. I. Khan \& M.R. Islam (Eds.), Petroleum Engineering Handbook: Sustainable Operations (pp. 243-293). Gulf Publishing Company. https://doi.org/10.1016/B978-1-933762-12-8.50014-3

Kuuskraa, V. A., \& Malone, T. (2016). $\mathrm{CO}_{2}$ enhanced oil recovery for offshore oil reservoirs. Anais do Annual Offshore Technology Conference, 4(May), $3764-3773$

Mattos, T. de M. (2018). Potencial de redução de emissões de gases de efeito estufa pela captura e sequestro de $\mathrm{CO}_{2}$ de destilarias de etanol do Centro Sul do Brasil para utilização em Recuperação Avançada de Petróleo [Dissertação de Mestrado, Universidade Federal do Rio de Janeiro]

Pant, D., Nadda, A. K., Pant, K. K., \& Agarwal, A. K. (2021). Advances in Carbon Capture and Utilization. In Energy, Environment, and Sustainability. https://doi.org/10.1007/978-981-16-0638-0_1

Patel, K., Shah, M., \& Sircar, A. (2018). Plasma Pulse Technology: An uprising EOR technique. Petroleum Research, 3(2), 180-188. https://doi.org/10.1016/J.PTLRS.2018.05.001

Perera, M. S. A., Gamage, R. P., Rathnaweera, T. D., Ranathunga, A. S., Koay, A., \& Choi, X. (2016). A Review of $\mathrm{CO}_{2}-$ Enhanced oil recovery with a simulated sensitivity analysis. Energies, 9(7). https://doi.org/10.3390/en9070481

Ravagnani, A. T. F. S. G., Ligero, E. L., \& Suslick, S. B. (2009). $\mathrm{CO}_{2}$ sequestration through enhanced oil recovery in a mature oil field. Journal of Petroleum Science and Engineering, 65(3-4), 129-138. https://doi.org/10.1016/j.petrol.2008.12.015 
Research, Society and Development, v. 11, n. 1, e16511124599, 2022

(CC BY 4.0) | ISSN 2525-3409 | DOI: http://dx.doi.org/10.33448/rsd-v11i1.24599

Ravagnani, A. T. F. S. G. (2007). Modelagem Técnico-Econômica de Sequestro de $\mathrm{CO}_{2}$ considerando Injeção em Campos Maduros [Tese de Doutorado, Universidade Estadual de Campinas]

Ritchie, H. \& Roser, M. (2020). "Energy". OurWorldInData.org. https://ourworldindata.org/energy

Sweatman, R., Crookshank, S., \& Edman, S. (2011). Outlook and technologies for offshore $\mathrm{CO}_{2}$ EOR/CCS projects. Anais do Offshore Technology Conference, 4(May), 2981-2993. https://doi.org/10.4043/21984-ms

Verma, M. K. (2015). Fundamentals of Carbon Dioxide-Enhanced Oil Recovery $\left(\mathrm{CO}_{2}\right.$-EOR)—A Supporting Document of the Assessment Methodology for Hydrocarbon Recovery Using $\mathrm{CO}_{2}$-EOR Associated with Carbon Sequestration. DC: US Department of the Interior, US Geological Survey, 19. https://doi.org/doi.org/10.3133/ofr20151071.

Xu, Y., Isom, L., \& Hanna, M. A. (2010). Adding value to carbon dioxide from ethanol fermentations. Bioresource Technology, 101(10), 3311-3319. https://doi.org/10.1016/j.biortech.2010.01.006. 